\title{
On Nonvanishing Univalent Functions with Real Coefficients ${ }^{\star}$
}

\author{
Wolfram Koepf \\ Freie Universität Berlin, Fachbereich Mathematik, Arnimallee 3, 1000 Berlin 33
}

Using the well-known Brickman representation for univalent functions, it is shown that the extreme points of the set $S_{0}(R)$ of nonvanishing univalent functions with real coefficients omit only real values. Furthermore a support point of $S_{0}(R)$ is shown to have the same property.

\section{Introduction}

Let $A$ be the set of analytic functions of the unit disk ID. $A$ is a locally convex linear space, so that the Krein-Mil'man theorem applies; i.e. the extreme points of a compact family $F$ span the closed convex hull: $\overline{c o} E F=\overline{c o} F$. For an introduction look for example in [5].

Recently Duren and Schober [4] examined the set $S_{0}$ of univalent functions which are normalized by the conditions

$$
f(0)=1, \quad 0 \notin f(\mathrm{D}) .
$$

$S_{0} \cup\{1\}$ is a compact subset of $A$. Duren and Schober had been interested in extreme points and support points of $S_{0}$. Recall that a support point of a family $F$ is a function which maximizes the real part of some continuous linear functional, that is not constant over $F$.

We shall give a characterization of the extreme points and support points of the subfamily $S_{0}(R)$ of nonvanishing univalent functions whose Taylor expansions at the origin have real coefficients.

\section{Extreme Points of $S_{0}(R)$}

Using the usual Brickman representation we get:

\footnotetext{
* This work is part of the author's doctoral thesis, accepted by the Department of Mathematics at the Free University of Berlin in 1984
} 
Lemma. Let be $f \in S_{0}(R)$. If $f$ omits some nonreal value a, then $f$ has a proper convex representation in $S_{0}(R)$.

Proof. Because $f$ is univalent and has real coefficients, the range of $f$ is symmetric with respect to the real axis, so that $f$ omits $\bar{a}$, too.

Because $a \notin \mathbb{R}$, we have now two omitted values which lie on an ellipse with foci in 0 and 1 , so that $f$ has the Brickman representation ([1], see [4], Theorem 1)

$$
f=t f_{1}+(1-t) f_{2}
$$

with $t \in] 0,1[$ and $(k=1,2)$

where

$$
f_{k}:=\psi_{k} \circ f \in S_{0}, \quad \psi_{1,2}(w):=\frac{w \pm \psi(w) \mp \psi(0)}{1+\psi(1) \mp \psi(0)}
$$

$$
\psi(w):=\sqrt{(w-a)(w-\bar{a})} .
$$

Now it remains to show that $f_{k}(k=1,2)$ or equivalently $\psi$ (expanded at the point 1) has real coefficients.

For $w \in \mathbb{R}$ one has $\psi(w) \in \mathbb{R}$, implying the result

Now we are able to state the following result about the extreme points.

Theorem 1. Every extreme point of $S_{0}(R) \cup\{1\}$ has the form

$$
\begin{array}{ll}
f(z)=\frac{(1+z)^{2}}{(1-y z)(1-\bar{y} z)}, & y \in \partial \mathbb{D} \backslash\{-1\} \quad \text { or } \\
f(z)=\frac{(1-z)^{2}}{(1-y z)(1-\bar{y} z)}, & y \in \partial \mathbb{I D} \backslash\{1\} .
\end{array}
$$

Proof. Because of the Lemma an extreme point of $S_{0}(R)$ omits only real values.

Thus with the origin all negative real numbers are omitted (because the range is simply connected). We next show that an extreme point of $S_{0}(R) \cup\{1\}$ omits no interval of the form $]-\infty, \varepsilon]$ for $\varepsilon>0$. In this case there would be a representation

$$
g=\frac{1}{1+\varepsilon} \cdot f+\frac{\varepsilon}{1+\varepsilon} \cdot 1
$$

with a certain function $f \in S_{0}$ with similar range. This is a representation within $S_{0} \cup\{1\}$, thus $g$ is not extreme.

So an extreme point omits $]-\infty, 0]$ and possibly a second real interval $[1+\varepsilon, \infty[, \varepsilon>0$. But the functions having this geometric property are exactly of the desired form.

\section{Support Points of $S_{0}(R)$}

Using the result about the extreme points we are able to give the following result about the support points of $S_{0}(R)$. 
Theorem 2. Every support point of $S_{0}(R)$ has the form

$$
f(z)=1+\kappa \frac{z}{(1-y z)(1-\bar{y} z)}
$$

for some $y \in \partial \mathbb{D}$ and $\kappa \in[-2(1-\operatorname{Re} y), 2(1+\operatorname{Re} y)], \kappa \neq 0$.

Proof. Let $L$ be a continuous linear functional over $A$ which is not constant within $S_{0}(R)$.

If $g$ is a support point of $S_{0}(R)$ with respect to $L$, we have

$$
M:=\operatorname{Re} L g=\max _{h \in S_{0}(R)} \operatorname{Re} L h .
$$

Because of Theorem 1 the function $g$ has the Choquet representation (see e.g. [5])

$$
g(z)=\int_{\partial \mathbb{D}} \frac{(1+z)^{2}}{(1-y z)(1-\bar{y} z)} d \mu_{+}(y)+\int_{\partial \mathbb{D}} \frac{(1-z)^{2}}{(1-y z)(1-\bar{y} z)} d \mu_{-}(y)
$$

with positive measures $\mu_{+}$and $\mu_{-}, \mu_{+}(\partial \mathrm{ID})+\mu_{-}(\partial \mathrm{ID})=1$, which are supported by the sets

$$
\left\{y \in \partial \mathbb{D} \mid \frac{(1 \pm z)^{2}}{(1-y z)(1-\bar{y} z)} \in E\left(S_{0}(R) \cup\{1\}\right)\right\}
$$

respectively.

Therefore it follows that $\mu_{+}$-a.e. and $\mu_{-}$-a.e. respectively

$$
\begin{aligned}
& \operatorname{Re} L\left\{\frac{(1+z)^{2}}{(1-y z)(1-\bar{y} z)}\right\}=M \text { and } \\
& \operatorname{Re} L\left\{\frac{(1-z)^{2}}{(1-y z)(1-\bar{y} z)}\right\}=M .
\end{aligned}
$$

Let $H_{ \pm}$be the subsets of $\partial \mathrm{ID}$ in which (2) hold. The functions $l_{ \pm}$defined by

$$
l_{ \pm}(y):=L\left\{\frac{(1 \pm z)^{2}}{(1-y z)(1-z / y)}\right\}
$$

are analytic in a neighborhood of $\partial \mathrm{ID}$.

Furthermore, let $g_{ \pm}(y):=\frac{1}{2}\left(l_{ \pm}(y)+\overline{l_{ \pm}(1 / \bar{y})}\right)$. Then $g_{ \pm}$is analytic in a neighborhood of $\partial \mathrm{ID}$ and $g_{ \pm}(y)=\operatorname{Re} l_{ \pm}(y)$ whenever $|y|=1$.

Assume now, for example $H_{+}$were infinite. Then $g_{+}$takes the value $M$ infinitely often in its domain of analycity and is thus constant, in particular $\operatorname{Re} l_{+}(y)=M$ whenever $|y|=1$.

But then we get substituting $y=-1$, that the constant function 1 is a support point with respect to $L$. Because of the representations

$$
1=\frac{1+z^{k}}{2}+\frac{1-z^{k}}{2}, \quad k \geqq 1
$$


it follows that $1 \pm z^{k}$ are support points with respect to $L$ for all $k \in \mathbb{N}$. Therefore the Toeplitz coefficients $b_{k}$ of $L$ (see e.g. [5], p. 36) vanish for all $k \in \mathbb{N}$.

Thus $L$ is constant in $S_{0}(R)$, which contradicts the assumption.

So $H_{+}$and - as a similar construction shows $-H_{-}$are finite, and (1) becomes a finite convex representation.

If it is a proper convex representation with a most two points, then the represented function $g$ is either multi-valued, because $g$ has poles on $\partial \mathrm{ID}$ of order at least 4 (see [2], p. 103), or $g$ is of the form $(t \in] 0,1[$ )

$$
g(z)=t \frac{(1+z)^{2}}{(1-y z)(1-\bar{y} z)}+(1-t) \frac{(1-z)^{2}}{(1-y z)(1-\bar{y} z)}
$$

which gives the desired result.

\section{Application to Other Normalizations}

Originally Brickman obtained a representation for the family $S$ of univalent functions, normalized by

$$
f(0)=0, \quad f^{\prime}(0)=1
$$

Because the construction is similar, our method works also in the family $S(R):=\{f \in S \mid f$ has real MacLaurin coefficients $\}$.

Corollary. Every extreme point of $S(R)$ is of the form

$$
f(z)=\frac{z}{(1-y z)(1-\bar{y} z)}, \quad|y|=1 .
$$

Proof. Using the Brickman representation in $S$ (see e.g. [3], Theorem 9.5) one gets similarly as in our Lemma, that an extreme point of $S(R)$ only omits real values, which is equivalent to representation (3).

We remark that this is a refinement of a result due to Brickman, MacGregor and Wilken [2], Theorem 4, who showed representation (3) for an extreme point of the closed convex hull of $S(R)$. Because of a general result due to Mil'man (see e.g. [5]), one knows a priori that

$$
E \overline{\operatorname{co}} S(R) \subset E S(R) \text {. }
$$

[2], Theorem 4, gives also a proof of the statement

$$
\{f \in S(R) \mid f \text { has representation (3) }\} \subset E \overline{c o} S(R),
$$

so that all families are equal:

$$
E \overline{\mathrm{CO}} S(R)=E S(R)=\{f \in S(R) \mid f \text { has representation (3) }\}
$$

Our method also applies to other normalizations, for example to the Montel classes with 


$$
f\left(z_{1}\right)=w_{1}, f\left(z_{2}\right)=w_{2}, \quad z_{1}, z_{2}, w_{1}, w_{2} \in \mathbb{R},
$$

because in this case there is a Brickman representation, too (see [5], Theorem 8.5). Thus here also the extreme points omit only real values.

\section{References}

1. Brickman, L.: Extreme points of the set of univalent functions. Bull. Am. Math. Soc. 76, 372-374 (1970)

2. Brickman, L., MacGregor, T.H., Wilken, D.R.: Convex hulls of some classical families of univalent functions. Trans. Am. Math. Soc. 156, 91-107 (1971)

3. Duren, P.L.: Univalent functions. Berlin-Heidelberg-New York-Tokyo: Springer 1983

4. Duren, P.L., Schober, G.: Nonvanishing univalent functions. Math. Z. 170, 195-216 (1980)

5. Schober, G.: Univalent functions-selected topics. Lecture notes 478, Berlin-Heidelberg-New York: Springer 1975

Received April 19, 1985 